Mat. Res. Bull., Vol. 29, pp. $31-40,1994$. Printed in the USA.

0025-5408/94 $\$ 6.00+.00$ Copyright (c) 1993 Pergamon Press Ltd.

\title{
A HYDROTHERMAL ROUTE FOR PRODUCTION OF DENSE, NANOSTRUCTURED Y-TZP
}

M.M.R. Boutz, R.J.M. Olde Scholtenhuis, A.J.A. Winnubst and A.J. Burggraaf University of Twente, Faculty of Chemical Technology, Laboratory for Inorganic Chemistry, Materials Science and Catalysis, P.O. Box 217, 7500 AE Enschede, The Netherlands.

(Received September 29, 1993; Communicated by N. Claussen)

\section{ABSTRACT}

Y-TZP powders were prepared either by calcination in air or crystallization under hydrothermal conditions of a hydrous gel, obtained by coprecipitation. Differences in powder properties, green compact structure and sinterability were examined. Crystallization under hydrothermal conditions occurs at temperatures as low as $190^{\circ} \mathrm{C}$ in the presence of ammonia. The hydrothermally treated powders are composed of soft agglomerates, that collapse under very low pressures, resulting in green bodies with high densities and small pore radii. The sinterability is greatly improved by the hydrothermal treatment and allowed the production of dense, nanostructured Y-TZP by free sintering at $1050^{\circ} \mathrm{C}$.

\section{MATERIALS INDEX: zirconia}

\section{Introduction}

Yttria stabilized tetragonal zirconia polycrystals (Y-TZP) is one of the most important ceramic materials for structural applications, due to its high strength and toughness (1). Y-TZP ceramics show true superplastic behaviour at elevated temperatures, if the grain size is below $1 \mu \mathrm{m}$. This has been demonstrated in 1986 for the first time by Wakai et al. (2), who obtained a tensile strain of $170 \%$ at $1450^{\circ} \mathrm{C}$. Such large tensile ductilities have made it possible to use superplastic forming techniques to fabricate complex shapes with good dimensional control $(3,4)$. Higher strain rates $(5)$ and larger elongations-to-failure (6) can be obtained by decreasing the grain size of the material.

The percentage of atoms residing in the grain boundary region becomes significant, once the grain size enters the nanometer-regime $(\leq 100 \mathrm{~nm})$. Novel material properties may arise for these nanostructured materials $(7,8)$. The evaluation of both mechanical properties (at low to moderate temperatures) and superplastic deformation behaviour (at elevated temperatures) requires the production of bodies with near-theoretical densities. However, preparing bulk materials with near-theoretical densities, while simultaneously 
keeping the grain size below $100 \mathrm{~nm}$, has proven to be quite difficult. The sinterability of the material should permit densification to proceed at such a low temperature, that only very limited grain growth occurs.

To obtain good sinterability at low temperatures ultra fine powders with a low degree of agglomeration are required. The compaction and sintering behaviour of such fine powders is determined to a large extent by the strength of their agglomerates. At a given crystallite size the agglomerate strength can be lowered by increasing the intraagglomerate porosity and/or decreasing the strength of the interparticle bonds (9).

Fine powders of $\mathrm{ZrO}_{2}$ solid solutions can be produced by a gel precipitation technique using metal chlorides as precursor chemicals $(10,11)$. This so-called chloride-method involves coprecipitation of metal hydroxides, followed by water/ammonia and ethanol washing steps. Subjecting the gel to ethanol washing is a crucial step to lower the strength of the agglomerates, since it doubles the intraagglomerate porosity after calcination compared to a gel that has only been treated with water/ammonia (12). Finally, the dried gel is calcined in air at $500-550^{\circ} \mathrm{C}$ to obtain a crystalline oxide. Inevitably, this calcination treatment leads to the formation of strong interparticle bonds. However, the intra-agglomerate porosity of these powders is sufficiently high to make the agglomerates collapse to a large extent under moderate confining pressures $(50-150 \mathrm{MPa})$.

Under hydrothermal conditions crystallization of $\mathrm{ZrO}_{2}$ solid solutions gels proceeds at temperatures well below those indicated above. Temperatures of $200-250^{\circ} \mathrm{C}$ have been reported for hydrothermal crystallization of $\mathrm{ZrO}_{2}-\mathrm{CaO}$ (13), $\mathrm{ZrO}_{2}-\mathrm{Y}_{2} \mathrm{O}_{3}$ (14) and $\mathrm{ZrO}_{2}-\mathrm{CeO}_{2}(15)$. Several investigations suggest that the formation of strong interparticle bonds under hydrothermal conditions is greatly diminished or even excluded.

Very limited work has been published so far, devoted to differences in sinterability of $\mathrm{ZrO}_{2}$ solid solutions prepared via hydrothermal crystallization or air calcination. In the case of $\mathrm{ZrO}_{2}-\mathrm{CaO}(13)$ and $\mathrm{ZrO}_{2}-\mathrm{CeO}_{2}(15)$ it has been observed that the sinterability is strongly improved by the hydrothermal treatment. In this paper Y-TZP powders have been prepared either by calcination in air or crystallization under hydrothermal conditions of a hydrous gel, obtained by coprecipitation. Differences in powder properties, green compact structure and sinterability have been examined. It has been investigated if dense, nanostructured Y-TZP can be prepared via the hydrothermal route.

\section{Experimental procedure}

\section{Powder synthesis}

The appropriate amounts of $\mathrm{ZrOCl}_{2} .8 \mathrm{H}_{2} \mathrm{O}$ (>99.5\% pure) and $\mathrm{YCl}_{3}$ (99.9\% pure) to prepare 5 mol\% $\mathrm{YO}_{1.5}$ stabilized TZP were dissolved in a $0.2 \mathrm{M} \mathrm{HCl}$ aqueous solution. This solution was added dropwise to an excess of a $25 \mathrm{wt} \%$ ammonia solution, while the $\mathrm{pH}$ was maintained above 11 . The obtained gel is then washed 8 times with water/ammonia mixtures, the ammonia concentration of which gradually decreased, to remove chlorine ions. Thereafter the gel was filtrated and divided into two parts. The first part was redispersed and washed twice in ethanol, dried at $120^{\circ} \mathrm{C}$ and finally calcined in static air at $500^{\circ} \mathrm{C}$ during 2 hours. This powder will be referred to as CA500. The second part has been used for hydrothermal crystallization. A stainless steel 
autoclave equipped with a $400 \mathrm{~cm}^{3}$ Teflon (polytetrafluorethylene) liner has been used for this purpose. Crystallization took place at $189-305^{\circ} \mathrm{C}$ during various times under $\mathrm{N}_{2}$ pressures of 80 - 90 bars. After the hydrothermal treatment the $\mathrm{pH}$ of the solution was 9 - 10. The slurry is then redispersed in ethanol, filtrated and dried at $120^{\circ} \mathrm{C}$.

Hydrothermally crystallized powders will be referred to as HCxyz, where xyz is the crystallization temperature $\left(\right.$ in ${ }^{\circ} \mathrm{C}$ ). As a final processing step, all powders were put on a rolling bench overnight in $100 \mathrm{ml}$ polyethylene bottles together with 3 Teflon marbles.

\section{Green compact formation and sintering}

Green compacts were prepared by cold isostatic pressing in two steps: first at 100 $160 \mathrm{MPa}$ and finally at $400 \mathrm{MPa}$. Sintering kinetics were analyzed by means of dilatometry using a Netzsch 402 E dilatometer. The dilatometer's sample thermocouple was carefully calibrated by the melting point method proposed by Henderson $e t$ al. (16). Densities were calculated from the green density and the observed linear shrinkage of the specimens, corrected for thermal expansion and weight loss (due to removal of absorbed water). Final densities calculated in this way were in good agreement with those measured by the Archimedes technique. All experiments were performed using cylindrical specimens with initial dimensions $7-15 \mathrm{~mm}$ (height) $\times 6-7$ $\mathrm{mm}$ (diameter).

Non-isothermal experiments were done by heating at $120^{\circ} \mathrm{C} / \mathrm{hr}$ to $1500^{\circ} \mathrm{C}$, immediately followed by cooling down at $300^{\circ} \mathrm{C} / \mathrm{hr}$. Isothermal experiments were done at 1000 and $1050^{\circ} \mathrm{C}$ during $5-20$ hours (heating rate $120^{\circ} \mathrm{C} / \mathrm{hr}$, cooling rate $300^{\circ} \mathrm{C} / \mathrm{hr}$ ).

\section{Characterization techniques}

A Stanton Redcroft DSC 1500 has been used for DSC analysis (in air, heating rate $600^{\circ} \mathrm{C} / \mathrm{hr}$ ). Nitrogen sorption isotherms were obtained at $77 \mathrm{~K}$ using a Micromeritics ASAP 2400 system. Specific surface areas were calculated by the BET-method. No corrections for microporosity were necessary. Mesopore size distributions have been calculated from the adsorption branch assuming a cylindrical pore shape. Bulk densities were measured by the Archimedes technique (in $\mathrm{Hg}$ ).

Crystallite sizes of the powders were determined by X-ray line broadening assuming Cauchy line profiles. Monoclinic volume contents have been calculated by the method proposed by Toraya (17). Impurity contents of powders were analyzed by AAS. Grain sizes in sintered specimens were determined by the lineal intercept technique from SEM (Hitachi S800) micrographs of polished, thermally etched cuts. Corrections for residual porosity were made by the method proposed by Wurst \& Nelson (18). The surfaces of the SEM-specimens were coated with $\mathrm{Au} / \mathrm{Pd}$ to prevent charging.

\section{$\underline{\text { Results }}$}

\section{Powder characteristics}

The characteristics of all 5 synthesized powder batches are summarized in Table I. XRD showed that all powders are crystalline and consist mainly out of the tetragonal phase. The monoclinic volume content of the hydrothermally treated powders increases with crystallization temperature and is higher than that of the air calcined CA500 powder 
batch.

A DSC-analysis has been performed in air of the CA500 gel after ethanol washing to determine the temperature, at which the amorphous $\rightarrow$ crystalline zirconia transition occurs. Two intense exothermic peaks, located at 315 and $450^{\circ} \mathrm{C}$, were observed in the DSC-trace. The first peak corresponds with the oxidative decomposition of chemisorbed ethoxygroups, while the second one coincides with the crystallization temperature (12). Crystallization in air will be further elaborated in the Discussion.

The specific surface area of the HC powders decreases with increasing crystallization temperature, caused by an increase in the average crystallite size. The surface area of the $\mathrm{HC} 189$ powder batch, crystallized at the lowest investigated temperature, is virtually identical to the one of the air calcined powder. The results of Table I demonstrate that Y-TZP powders with surface areas $>100 \mathrm{~m}^{2} / \mathrm{gr}$ can be prepared by hydrothermal methods.

Assuming that only the intra-agglomerate porosity $(\mathrm{P})$ consists out of mesopores, $\mathrm{P}$ can be calculated from the $\mathrm{N}_{2}$ sorption isotherms of the various powders using:

$$
P=\frac{V_{p} \rho_{t h}}{\left(V_{p} \rho_{t h}+1\right)}
$$

where $\mathrm{V}_{\mathrm{p}}$ is the total mesopore volume in $\mathrm{cm}^{3} / \mathrm{gr}$, determined from the adsorption branch of the hysteresis, and $\rho_{\mathrm{th}}$ is the theoretical density $\left(6.06 \mathrm{gr} / \mathrm{cm}^{3}\right)$. In Table I it can be seen that the intra-agglomerate porosities are in the range $60-67 \%$ for all powders. The average pore widths range from 11 to $15 \mathrm{~nm}$ for the various powders. Considering the size of the crystallites $(7-9 \mathrm{~nm})$, this justifies the assumption that the measured mesoporosity is located solely within the agglomerates.

AAS-analysis revealed that the cationic impurity content was similar for all powders. Typical detected concentrations were (in wt \%): $.02 \mathrm{Al}, .01 \mathrm{Fe}, .002 \mathrm{~K}$ and .002 $\mathrm{Na}$. The chlorine content of the CA500 powder batch $(.01 \mathrm{wt} \%)$ is one order of magnitude higher compared to the $\mathrm{HC}$ materials.

\section{Green compact formation}

Differences in compaction behaviour are quite significant comparing the air calcined powder batch with the hydrothermally treated ones. During cold isostatic compaction relative densities of $47 \%$ are already obtained at $180 \mathrm{MPa}$ for the $\mathrm{HC}$ materials, while only $29 \%$ relative density is observed for the air calcined one. At the highest applied pressure (400 MPa) the green compacts of the HC powders are $53-54 \%$ dense, compared to only $45 \%$ for the CA500 powder. At compaction pressures below 160 $\mathrm{MPa}$ green compacts of the HC powders did not remain intact, presumably due to a lack of sufficient mechanical interlocking. This prevented the determination of agglomeratestrength from a compaction curve for the HC powders.

Figure 1 shows the sorption isotherms of green compacts (pressed at $400 \mathrm{MPa}$ ) of CA500 and HC189. The sorption isotherms of all 4 investigated HC materials are very similar. The shape of the hysteresis loop is an indication for the pore morphology. For the $\mathrm{HC}$ materials the hysteresis is predominantly of type $\mathrm{E}$, while it is of a mixed type A/E for the air calcined material, following the classification of de Boer (19). Type $E$ is indicative of tubular capillaries with wide parts of various widths, while type A corresponds with a cylindrical pore shape. During sintering the hysteresis shape of TZP 


\section{TABLE I}

Characteristics of the investigated powders, calcined in air at $500^{\circ} \mathrm{C}$ (CA500) or hydrothermally crystallized at $189-305^{\circ} \mathrm{C}$.

\begin{tabular}{||l|c|c|c|c|c|c||}
\hline \hline $\begin{array}{l}\text { Material } \\
\text { code }\end{array}$ & $\begin{array}{c}\mathrm{T}_{\text {hydro }} \\
{\left[{ }^{\circ} \mathrm{C}\right]}\end{array}$ & $\begin{array}{c}\mathrm{t}_{\text {hydro }} \\
{[\mathrm{min}]}\end{array}$ & $\begin{array}{c}\mathrm{S}_{\text {BET }} \\
{\left[\mathrm{m}^{2} / \mathrm{gr}\right]}\end{array}$ & $\begin{array}{c}\mathrm{D}_{\text {XRLB }} \\
{[\mathrm{nm}]}\end{array}$ & $\begin{array}{c}\mathrm{V}_{\mathrm{m}} \\
{[\%]}\end{array}$ & $\begin{array}{c}\mathrm{P} \\
{[\%]}\end{array}$ \\
\hline CA500 & - & - & 113 & 7 & 12 & 67 \\
\hline HC189 & 189 & 80 & 110 & 7 & 18 & 60 \\
\hline HC254 & 254 & 210 & 102 & 7 & 20 & 63 \\
\hline HC269 & 269 & 290 & 97 & 8 & 28 & 63 \\
\hline HC305 & 305 & 224 & 83 & 9 & 38 & 66 \\
\hline
\end{tabular}

$T_{\text {hydro }}$ : temperature of hydrothermal treatment, $t_{\text {hydro }}$ : duration of hydrothermal treatment, $\mathrm{S}_{\mathrm{BET}}$ : BET surface area, $\mathrm{D}_{\mathrm{XRLB}}$ : crystallite size as determined by $\mathrm{X}$-ray line broadening, $\mathrm{V}_{\mathrm{m}}$ : monoclinic volume content, $P$ : intra-agglomerate porosity.

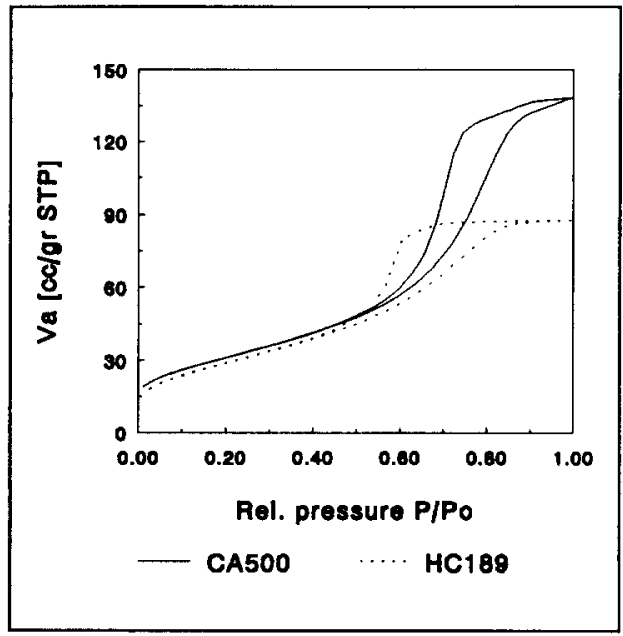

FIG. 1

$\mathrm{N}_{2}$ sorption isotherms of green compacts of the air calcined CA500 and the hydrothermally crystallized HC189 material.

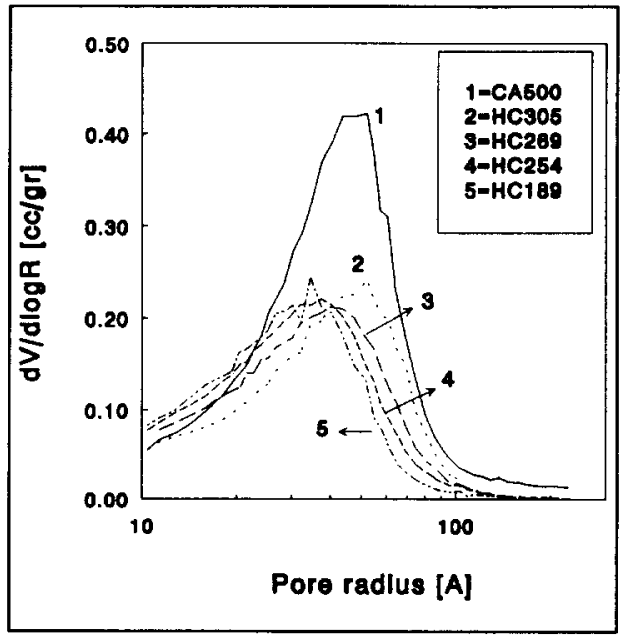

FIG. 2

Pore size distributions of green compacts of the 5 investigated materials.

compacts becomes more and more of type A due to progressive neck formation (20). The differences in green porous texture between the air calcined and the hydrothermally treated materials are therefore most probably caused by partial sintering already taking 
place during calcination in air of the hydroxide gel, while this is not the case under hydrothermal conditions.

The pore size distributions of green compacts pressed at $400 \mathrm{MPa}$ of all 5 materials can be found in fig.2. The most striking observation is that the fraction of pores with radii exceeding $3.5 \mathrm{~nm}$ is strongly diminished in green compacts of the hydrothermally treated powders compared to the air calcined one. The average pore radius $R_{p}$ of the HC compacts increases with increasing crystallization temperature: $R_{p}$ equals $2.4 \mathrm{~nm}$ for $\mathrm{HC} 189$ and increases to $3.0 \mathrm{~nm}$ for HC305. The largest average pore radius is observed in the CA500 material: $3.3 \mathrm{~nm}$. Since the crystallite sizes of the hydrothermally treated powders are larger than that of the air calcined powder batch (see Table I), the smaller average pore radii of the $\mathrm{HC}$ green compacts result from improved compactability resulting in a better particle stacking.

\section{Sintering}

The increase of relative density with temperature during heating at $120^{\circ} \mathrm{C} / \mathrm{hr}$ to $1500^{\circ} \mathrm{C}$, as measured by dilatometry, is given in fig. 3 for CA500, HC189 and HC254. A great difference in sinterability is observed: the hydrothermally treated materials are already $95 \%$ dense at $1150^{\circ} \mathrm{C}$, whereas a temperature of $1350^{\circ} \mathrm{C}$ is required for the air calcined material to reach the same density. After this sintering schedule all materials are $96 \%$ dense. XRD showed only diffraction lines characteristic of the tetragonal phase after this heat treatment for all materials.

From fig. 3 it is evident that it should be possible to sinter the HC materials to full density at temperatures below $1100^{\circ} \mathrm{C}$. Isothermal sintering experiments were therefore performed at 1000 and $1050^{\circ} \mathrm{C}$. The increase in relative density with time during sintering at $1050^{\circ} \mathrm{C}$ is illustrated in fig. 4 for 4 materials. The HC materials are $94-96 \%$ dense after sintering for 5 hours at this very low temperature, whereas the air calcined one reaches only $72 \%$ relative density after 10 hours. It can be seen in fig. 4 that only 1.5 hours at $1050^{\circ} \mathrm{C}$ are needed to reach $95 \%$ relative density for the $\mathrm{HC} 189$ material.

Sintering experiments were also performed at $1000^{\circ} \mathrm{C}$ with the $\mathrm{HC}$ materials. From these tests (not shown) it could be concluded that the sinterability of the HC materials increases with decreasing crystallization temperature: final densities of 88,92 and $93 \%$ were observed after 20 hours for respectively material HC305, HC269 and HC254.

\section{Microstructures after sintering at $1050^{\circ} \mathrm{C}$}

Microstructures after sintering at $1050^{\circ} \mathrm{C}$ were examined by SEM. The average grain size of the hydrothermally treated materials after sintering at $1050^{\circ} \mathrm{C}$ during 5 or 10 hours equals respectively 95 and $100 \mathrm{~nm}$. It was furthermore observed that the residual porosity in the CA500 material ( $72 \%$ dense) consists mainly out of pores having widths larger than the average grain size, while very few of such pores were observed in the hydrothermally treated materials (94-96\% dense). This is illustrated clearly in fig.5, where the microstructure after sintering at $1050^{\circ} \mathrm{C}$ during 10 hours is shown for the CA500 and HC189 material. 


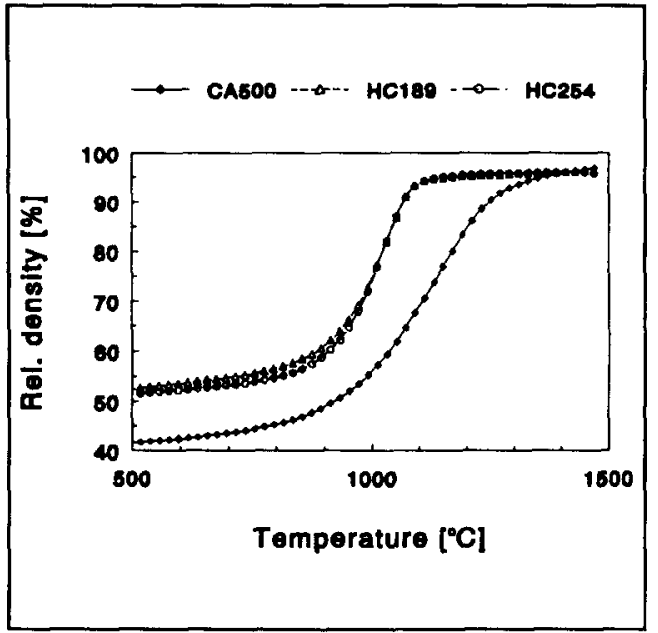

FIG. 3

Increase of relative density with temperature during heating at $120^{\circ} \mathrm{C} / \mathrm{hr}$.

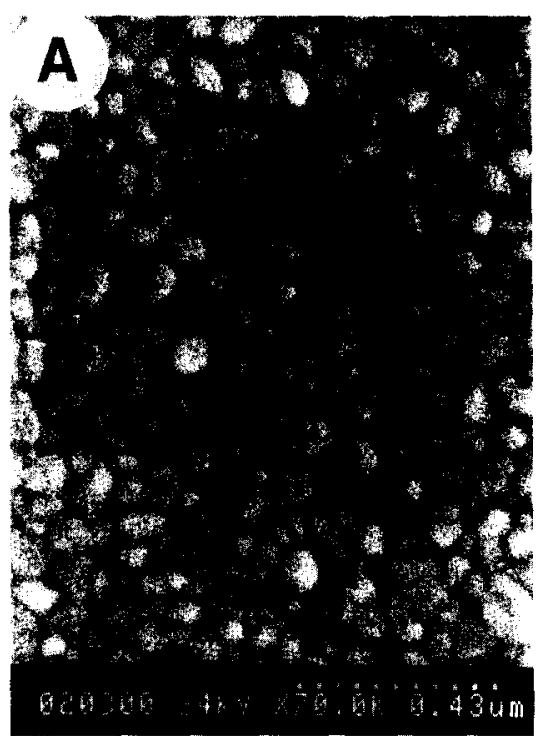

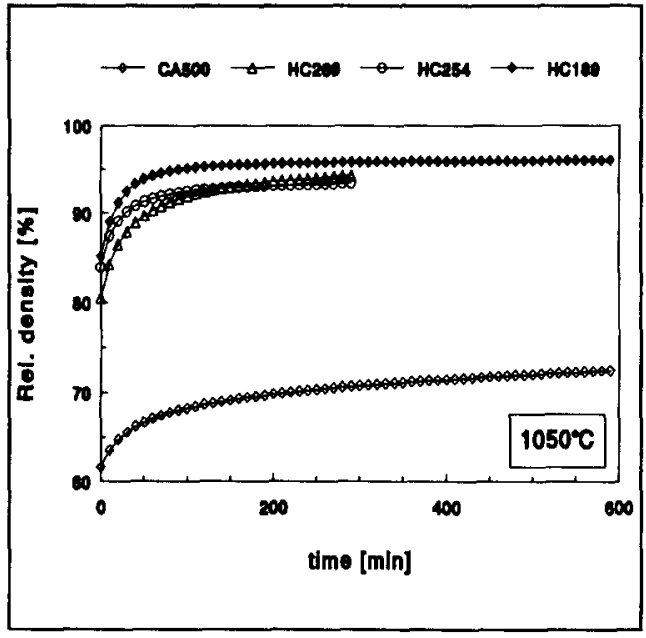

FIG. 4

Increase of relative density with time during sintering at $1050^{\circ} \mathrm{C}$.

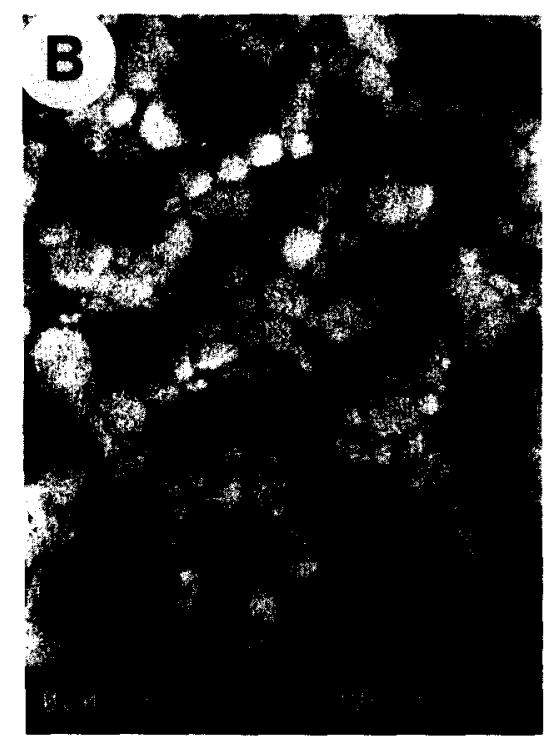

FIG. 5

Microstructure after sintering at $1050^{\circ} \mathrm{C}$ during 10 hours of the (a) $\mathrm{HC} 189$ and (b) CA500 material. 


\section{Discussion}

DSC-analysis indicated that the amorphous $\rightarrow$ crystalline transition occurs near $450^{\circ} \mathrm{C}$ in air for $5 \mathrm{~mol} \% \mathrm{YO}_{1.5}$ stabilized zirconia. However, the relatively high heating rate $\left(600^{\circ} \mathrm{C} / \mathrm{hr}\right)$ used in this analysis leads to an overestimation of the crystallization temperature. Theunissen (21) used a high temperature Guinier camera for XRD (heating rate $15^{\circ} \mathrm{C} / \mathrm{hr}$ ) and observed the first appearance of diffraction lines of the tetragonal phase at $400^{\circ} \mathrm{C}$. Livage et al. (22) found from a series of isothermal experiments that the minimum temperature required for complete crystallization of pure $\mathrm{ZrO}_{2}$ in air equals $350^{\circ} \mathrm{C}$. It can thus be concluded, that temperatures of $350-400^{\circ} \mathrm{C}$ are needed for complete crystallization of zirconia in air.

It has been observed in this investigation, that under hydrothermal conditions crystallization occurs already at temperatures as low as $190^{\circ} \mathrm{C}$. Nishizawa et al. (23) demonstrated that the crystallization process under hydrothermal conditions depends strongly on concentration and type of mineralizer. They observed that undoped zirconia crystallizes at $260^{\circ} \mathrm{C}$ in pure water, whereas a temperature of only $130^{\circ} \mathrm{C}$ is required in a $1 \mathrm{M} \mathrm{NaOH}$ solution. The presence of $\mathrm{NH}_{4} \mathrm{OH}$ in the gels used here for hydrothermal treatments is therefore most likely responsible for the low crystallization temperature.

It has furthermore been found that the compactability of the hydrothermally treated powders is strongly improved compared to the air calcined powder batch. As mentioned in the introduction, the compactability of fine powders is largely determined by the strength of the agglomerates. Apparently, the HC powders are composed of much weaker agglomerates than the calcined one. Since the intra-agglomerate porosities of all investigated powders are quite similar, differences in agglomerate strength must be related to weaker interparticle bonds in the HC powders. Recently, a new series of $\mathrm{Y}$ TZP powder batches has been prepared via hydrothermal crystallization at $195^{\circ} \mathrm{C}$ or calcination in air at $500^{\circ} \mathrm{C}$. Compaction curves could be determined for both types of powders, which permitted the determination of agglomerate-strengths. The agglomeratestrength of the air calcined powder $(110 \mathrm{MPa})$ was observed to be much higher than the one of the $\mathrm{HC}$ powder $(<15 \mathrm{MPa})$. These findings are in very good agreement with those of Haberko \& Pyda (13), who studied hydrothermal crystallization of $\mathrm{ZrO}_{2}-\mathrm{CaO}$.

The temperature needed for crystallization is probably one of the key parameters in determining interparticle bond strength. During calcination in air at $500^{\circ} \mathrm{C}$ or more, partial sintering causes the formation of strong interparticle bonds, while this is not or much less the case at the very low temperatures needed for hydrothermal crystallization.

At the elevated $\mathrm{pH}$ conditions used here for hydrothermal treatments $(\mathrm{pH}=9-10)$ crystallization of the hydrous gel takes place in situ via a solid state transformation, because the suspensions are undersaturated with respect to the solubility of zirconiumhydroxides at $25^{\circ} \mathrm{C} \mathrm{(24).} \mathrm{The} \mathrm{isoelectric} \mathrm{point} \mathrm{of} \mathrm{Y-TZP} \mathrm{is} \mathrm{situated} \mathrm{near} \mathrm{pH} 7$. The tetragonal zirconia particles will therefore posses a negative surface charge during the hydrothermal treatment. The resulting electrostatic repulsion between the particles will decrease the extent of agglomeration and thus prevent the formation of strong interparticle bonds.

More homogeneous green bodies with higher green densities and smaller average pore radii are made with the HC powders. This leads to a strongly improved sinterability of the HC materials and has made it possible to produce dense $(94-96 \%)$ Y-TZP with grain sizes of $95-100 \mathrm{~nm}$ by free sintering at $1050^{\circ} \mathrm{C}$. The air calcined material reaches densities above $95 \%$ during free sintering only at $1150^{\circ} \mathrm{C}$, the resultant grain size being 
near $200 \mathrm{~nm}$. Boutz et al. (25) have shown recently that only by sinter forging at $1100^{\circ} \mathrm{C}$ under a large compressive stress of $84 \mathrm{MPa}$ can a density of $93 \%$ with an average grain size of $100 \mathrm{~nm}$ be obtained with an air calcined Y-TZP material.

Theunissen et al. (26) reported the production of dense (97\%) Y-TZP with a grain size below $100 \mathrm{~nm}$ by free sintering at $1050^{\circ} \mathrm{C}$ for an air calcined powder. The followed powder synthesis route, however, yields strongly fluctuating results. Currently, all the different steps of this synthesis route are being evaluated and optimized to enhance reproducibility. From recently performed experiments a good reproducibility has been found for the hydrothermal route in producing dense, nanostructured Y-TZP.

\section{Conclusions}

Crystallization of Y-TZP occurs under hydrothermal conditions at temperatures as low as $190^{\circ} \mathrm{C}$ in the presence of ammonia, whereas a temperature of $400^{\circ} \mathrm{C}$ is needed for crystallization in air. High surface area $\left(>100 \mathrm{~m}^{2} / \mathrm{gr}\right.$ ) powders composed of weak agglomerates, that collapse under very low pressures, are produced via this hydrothermal route. Homogeneous green bodies with high densities and small pore radii made with the HC powders lead to a strongly improved densification behaviour compared to the air calcined material. In this way dense Y-TZP with grain sizes below $100 \mathrm{~nm}$ could be prepared by free sintering at $1050^{\circ} \mathrm{C}$. This offers the exciting opportunity to investigate for the first time the mechanical properties and superplastic deformation of this nanostructured material.

\section{Acknowledgements}

Akzo Chemicals bv is greatfully acknowledged for financial support of this investigation.

\section{$\underline{\text { References }}$}

1. M.V. Swain and L.R.F. Rose, J. Am. Ceram. Soc. 69 [7], 511 (1986).

2. F. Wakai, S. Sakaguchi and Y. Matsuno, Adv. Cer. Mater. 1 [3], 259 (1986).

3. X. Wu and I-W. Chen, J. Am. Ceram. Soc. 73 [3], 746 (1990).

4. J. Wittenauer, T.G. Nieh and J. Wadsworth, Scr. Metall. Mater. $\underline{26}, 551$ (1992).

5. M.M.R. Boutz, Ph.D. thesis, Chapter IV, University of Twente, 1993.

6. T.G. Nieh and J. Wadsworth, Scr. Metall. Mater. 24,763 (1990).

7. A.J. Burggraaf, J. Eur. Ceram. Soc. $\underline{9}, 245$ (1992).

8. A.J. Burggraaf, A.J.A. Winnubst and H. Verweij, to be published in the Proceedings of the third Meeting of the European Ceramic Society, Madrid, September 12-17, 1993.

9. H. Rumf, Chem. Ing. Technol. $\underline{30}, 144$ (1958).

10. K. Haberko, Ceramurgia Int. $\underline{5}$ [4], 148 (1979).

11. W.F.M. Groot Zevert, G.S.A.M. Theunissen, A.J.A. Winnubst and A.J. Burggraaf, J. Mater. Sci. 25, 3449 (1990).

12. P.D.L. Mercera, J.G. van Ommen, E.B.M. Doesburg, A.J. Burggraaf and J.R.H. Ross, J. Mater. Sci. $\underline{27}, 4890$ (1992). 
13. K. Haberko and W. Pyda, Advances in Ceramics, Vol. 12, p. 774, American Ceramic Society (1984).

14. W. Pyda, K. Haberko and M.M. Bucko, J. Am. Ceram. Soc. 74 [10], 2622 (1991).

15. T. Sato, K. Dosaka, T. Yoshioka, A. Okuwaki, K. Torii and Y. Onodera, J. Am. Ceram. Soc. 75 [3], 552 (1992).

16. J.B. Henderson, W.D. Emmerich and E. Wassmer, J. Thermal Anal. $\underline{\text { 32, }} 1905$ (1987).

17. H. Toraya, M. Yoshimura and S. Somiya, J. Am. Ceram. Soc. $\underline{67}$, C119 (1984).

18. J.C. Wurst and J.A. Nelson, J. Am. Ceram. Soc. $\underline{55}$, 109 (1972).

19. J.H. de Boer, The structure and properties of porous materials, p. 68, Butterworth scientific publications (1958).

20. M.M.R. Boutz, A.J.A. Winnubst and A.J. Burggraaf, submitted to J. Eur. Ceram. Soc.

21. G.S.A.M. Theunissen, Ph.D. thesis, Chapter II, University of Twente, 1991.

22. J. Livage, K. Doi and C. Mazières, J. Am. Ceram. Soc. $\underline{51}$ [6], 349 (1968).

23. H. Nishizawa, N. Yamasaki, K. Matsuoka and H. Mitsushio, J. Am. Ceram. Soc. $\underline{65}$ [7], 343 (1982).

24. J.H. Adair, R.P. Denkewicz and F.J. Amagada, Ceramic Powder Science II, p. 135, The American Ceramic Society, Westerville, Ohio (1988).

25. M.M.R. Boutz, A.J.A. Winnubst, A.J. Burggraaf, M. Nauer and C. Carry, Proceedings of Zirconia $\mathrm{V}$, in press.

26. G.S.A.M. Theunissen, A.J.A. Winnubst and A.J. Burggraaf, J. Eur. Cer. Soc. 11, 315 (1993). 